\title{
Prevalence of Human papillomavirus (HPV) genotypes in Ecuadorian women.
}

Abstract: Infection with the human papillomaviruses (HPV) is now well-established as the leading cause in the development of cancer of the uterine cervix. HPV 16 and 18 are described as the most prevalent high-risk genotypes, involving about $70 \%$ of registered cervical cancer cases worldwide. Among women in Ecuadorian population, cervical cancer is the second most frequent malignancy, and about 75\% of active sexual women will be infected with at least one type of HPV genotypes throughout their lives. In the present investigation, the prevalence of HPV genotypes was determined from samples collected at Society for the Fight Against Cancer (SOLCA) in Ecuadorian women between 18 to 78 years. The participants underwent a Pap test, biopsy, cervical and vaginal swab for HPV detection and genotype identification. The results show the high prevalence index of HPV genotype 16 and 58, with a higher burden from the second to the fourth decade of life.

KeyWords: Human papillomavirus, HPV 16 and 18, cervical cancer, Ecuadorian women.

\section{Introduction}

Epidemiological studies have established human papillomavirus (HPV) infection as the primary cause of invasive cervical cancer (ICC) and a relevant factor in other anogenital diseases ${ }^{1,2}$. Cervical cancer is the second most common cancer and is now the first leading cause of cancer deaths in South America women².

HPV genome is a circular double-stranded DNA molecule of 8000 base-pair, which contains an average of eight open reading frames, divided into three regions. The first is the upstream regulatory region (URR), which has the regulatory function of the transcription of the viral genes E6 and E7. The second is an Early region, which consists of six open reading fragments (E1, E2, E4, E5, E6, and E7), which code for nonstructural proteins involved in replication and oncogenesis, but the central molecules in the replication process are the viral proteins $\mathrm{E} 6$ and $\mathrm{E} 7$, which interact with host cell proteins to induce proliferation and malignant transformation of cells ${ }^{3}$. The third is the late region, which encodes the structural proteins $\mathrm{L} 1$ and $\mathrm{L} 2^{4}$.

Over 230 HPV genotypes have been identified, and about 40 of them can infect the anogenital and mucosal region ${ }^{5,6}$. Genital HPV types have been classified into high oncogenic risk groups usually associated with ICC and low oncogenic risk groups which is found mainly in genital warts. However, there are discrepancies related to the categorization of many HPV types with possible oncogenic risk? Nowadays, the International Agency for Research on Cancer (IARC) only recognize 12 genotypes $(16,18,31,33,35,39,45,51,52,56$, $58,59)$ as high risk, from which HPV 16 and 18 are the most prevalent worldwide, involving about $70 \%$ of cervical cancer cases $^{5}$.

The HPV infection is highly transmissible, usually by skin to skin contact, although not necessarily sexual intercourse ${ }^{8}$. This kind of disease can occur at any age, and it is estimated that more than $50-75 \%$ of sexually active women are infected with at least one HPV genotype throughout their ${ }^{6,9}$. Regarding Ecuador, there is no data published by $\mathrm{PAHO} / \mathrm{WHO}$ on the prevalence and incidence of HPV. Nevertheless, according to the National Institute of Statistics and Censuses in Ecuador (INEC), cervical cancer is the second most common malignancy with a prevalence in younger women than 35-44 years of age, declining in elderly women ${ }^{7,8}$.

The objective of this work is to estimate the prevalence of high-risk HPV genotypes in Ecuadorian women, focusing on genotypes 16 and 18 associated with cervical cancer.

\section{Clinical samples}

The criteria used for the clinical sample was: adult women between 18 - 78 years, born and living in Ecuador from different urban areas (table 1$)^{7,8}$. The samples were collected at Society for the Fight Against Cancer (SOLCA for its abbreviation, Spanish) Hospital from women diagnosticated HPV, cervical cancer, cervicitis, cervical dysplasia or without any cervical problem ${ }^{5,8-10}$.

The women participated in two procedures, including their clinic history through a survey (age, marital status, pregnancies, smoking, previous diagnoses, previous Pap test and number of sexual partners), and physical exams for sample collection $^{5}$. The methods used for sample collection were: Pap test, vaginal swab, cervical swab and biopsy (Table 1). The Pap test, also called Pap smear, consists of a speculum that is inserted into the vagina, and spatula or brush will scrape cell samples from the cervix $x^{5,8,9}$. The vaginal and cervical swab method uses three cotton swabs that are rubbed simultaneously in the area of vagina and cervix while the speculum is inside of the vagi$n a^{5}$. The last way is the biopsy, which consists of removing a sample of tissue from the lower part of the uterus with laser or scalpel. All the samples were analyzed in different laboratories within the country and abroad.

\section{Molecular analysis}

The analysis of the samples collected allows to knows the prevalence of HPV and its different genotypes in Ecuadorian women. There are three molecular analysis: DNA extraction, HPV detection, and genotype identification. The various techniques for DNA extraction and purification were Cetyl Trimethyl Ammonium Bromide (CTAB) method, spectrophotometry or commercial methods such as AmpliLute Liquid Media Extraction Kit or kit PVH Fast 2.08-10. The detection and genotyping of HPV were made by conventional PCR, reverse blood strip, genotyping method, linear array 
HPV genotyping test and commercial methods such as HPV GenoArray Diagnostic Kit ${ }^{5,8-10}$.

The assays made focuses on the characterization of forty (40) types of HPV that produce infection in the genital tract, especially, on the twelve (12) genotypes considered of high risk because of cause cervical cancer, including 16, 18 y 58.

\section{HPV prevalence}

A total of 6 research included in this study. Table 1 shows the percentage and numerical amounts of HPV prevalence of each population, which demonstrates that most of the research exceeds $50 \%$ of women with positive HPV.

HPV 16, 58 and 66 were the most commonly detected type (Table 2). In many cases, multiple HPV genotypes were identified, including high and low-risk genotypes.

\section{Conclusions}

The overall frequency of high-risk HPV genotypes detected in women population was high for HPV 16 and 58 and low for HPV 18 in Ecuador. Moreover, evidence was found of a high prevalence rate of HPV 66 of low-risk considered as possible oncogenic risk genotype. The data are very similar to those obtained around the world, suggesting once again that strategies on sexual education and prevention methods in adolescents could reduce the incidence and mortality rate of cervical cancer in the Ecuadorian population.

Ecuador is among countries with the highest incidence of cervical cancer in Latin America with Colombia, Brazil, and Peru'. According to INEC in 2017, 755 deaths for cervical cancer were recorded. Therefore, HPV vaccination is the best

\begin{tabular}{|c|c|c|c|c|c|}
\hline \multicolumn{2}{|c|}{ Population } & \multicolumn{1}{c}{ HPV +} & Prevalence & Age & Collection Methods \\
\hline $1^{5}$ & 1,581 & 689 & $43 \%$ & $20-70$ & Pap test \\
\hline $2^{8}$ & 124 & 84 & $68 \%$ & $18-55$ & Pap test / Biopsy \\
\hline $3^{9}$ & 302 & 73 & $24 \%$ & $18-78$ & Pap test / vaginal swab /cervical swab \\
\hline $4^{10}$ & 164 & 141 & $86 \%$ & $19-77$ & Biopsy \\
\hline $5^{11}$ & 63 & 55 & $87 \%$ & - & Biopsy \\
\hline $6^{12}$ & 166 & 113 & $68 \%$ & - & Cervical swab / Biopsy \\
\hline
\end{tabular}

Table 1. Numerical and percentage prevalence of HPV, range of ages of each population and methods of collection of clinical samples.

Table 2. Prevalence of HPV genotypes 16, 18, 58 and 66 in Ecuadorian women.

\begin{tabular}{|cccccccccc|}
\hline & Gen 16 & $\%$ & Gen $\mathbf{1 8}$ & $\%$ & Gen 66 & $\%$ & Gen $\mathbf{5 8}$ & $\%$ \\
\hline $1^{5}$ & 87 & $5.5 \%$ & 50 & $3.1 \%$ & 41 & $2.6 \%$ & 32 & $2 \%$ \\
\hline $2^{8}$ & 3 & $2.4 \%$ & 1 & $0.8 \%$ & 6 & $4.8 \%$ & 1 & $0.8 \%$ \\
\hline $3^{9}$ & 7 & $2.3 \%$ & 1 & $0.3 \%$ & 1 & $0.3 \%$ & 6 & $2 \%$ \\
\hline $4^{10}$ & 59 & $35.9 \%$ & 4 & $2.4 \%$ & 11 & $7.8 \%$ & 43 & $30.5 \%$ \\
\hline $5^{11}$ & 12 & $31.7 \%$ & 2 & $4.9 \%$ & 5 & $12.2 \%$ & 20 & $48.8 \%$ \\
\hline $6^{12}$ & 39 & $23.5 \%$ & 4 & $2.4 \%$ & 1 & $0.6 \%$ & 18 & $10.8 \%$ \\
\hline
\end{tabular}

\section{Discussion}

There are few studies about the prevalence of HPV and its different genotypes in Ecuadorian women. Nevertheless, the data collected showed considerable evidence of the presence of HPV in participants because the incidence is approaching or exceeding $50 \%$ in most of the studies.

Based on the results, the twelve genotypes of HPV consider of high risk are present in Ecuadorian women, including genotype 16, 18, and 58 that appear in all research. Most of these genotypes are associated with cervical problems such as cervical dysplasia or cervicitis, and cancer. Each high-risk genotype can produce different cervical risks and can act independently or with multiple genotypes. In most cases, numerous HPV genotypes were detected between low and high risk increasing the probability of developing cervical injuries.

HPV genotypes 16, 18, and 58 are considered the most common high-risk genotypes, so, genotype 16 , a viral type with the strong transmission, persistence, and transformation, has a high prevalence index in the studies, followed by genotype 58 considered as oncogenic. However, genotype 18 had a low frequency which is in concordance with previous findings showing low percentages in South America (5\% of HPV infections) compared with North America (11\%) and Europe (8\%). The reason of these differences in geographic distribution of HPV genotypes is unknown, however various authors have proposed complex interactions between HPV types and HLA haplotypes as a possible explanation ${ }^{10}$.

Other criteria were the age that showed HPV prevalence in women between 20 - 40 years during their active sexual life. On the other hand, the genotype 66, into the category of low risk, was the most prevalent gen of low risk in Ecuador. strategy to prevent and protect Ecuadorian women from ICC. In Ecuador, this vaccine is freely distributed by "Ministerio de Salud Pública".

Besides, the American Cancer Society recommends annual tests such as Pap test, vaginal, and cervical swab, especially in women over 20 years of sexually active and HPV DNA test for women over 30 years.

\section{Bibliographic references}

1. Clifford GM, Smith JS, Plummer M, Muñoz N, Franceschi S. Human papillomavirus types in invasive cervical cancer worldwide: A meta-analysis. Br J Cancer. 2003;88(1):63-9.

2. HPV Centre. Human Papillomavirus and Related Diseases Report. HPV Inf Cent. 2014;(Afghanistan):iii.

3. Muñoz N, Castellsagué $X$, de González AB, Gissmann L. Chapter 1: HPV in the etiology of human cancer. Vaccine. 2006;24(SUPPL. 3):1-10.

4. Beltrán JF. Aspectos generales sobre la estructura y función de las proteínas codificadas por el virus del Papiloma Humano. Rev CENIC Ciencias Biol. 2014:45(2):108-18.

5. García Muentes GD, García Rodríguez LK, Burgos Galarraga RI, Almeida Carpio F, Ruiz Cabezas JC. Genotypes distribution of human papillomavirus in cervical samples of Ecuadorian women. Rev Bras Epidemiol. 2016;19(1):160-6.

6. Dalgo Aguilar P, Loján González C, Córdova Rodríguez A, Acurio Paéz K, Arévalo AP, Bobokova J. Prevalence of High-Risk Genotypes of Human Papillomavirus: Women Diagnosed with Premalignant and Malignant Pap Smear Tests in Southern Ecuador. Infect Dis Obstet Gynecol. 2017;2017:12-4.

7. Brown CR, Leon ML, Muñoz K, Fagioni A, Amador LG, Frain B, et al. Human papillomavirus infection and its association with cervical dysplasia in Ecuadorian women attending a private cancer screening clinic. Brazilian J Med Biol Res. 2009;42(7):629-36. 
8. Muñoz N, Bosch FX, de Sanjosé S, Herrero R, Castellsagué $X$ Shah K V., et al. Epidemiologic Classification of Human Papillomavirus Types Associated with Cervical Cancer. N Engl J Med. 2003;348(6):518-27.

9. González-Andrade F, Sánchez D. HPV genotyping in anogenital abnormal samples of Ecuadorian women. Cancer Biomarkers. 2009;5(4-5):225-32.

10. Mejía L, Muñoz D, Tinoco L, Zapata S. Prevalence of Human Papillomavirus Types in Cervical Cancerous and Precancerous Lesions of Ecuadorian Women. J Med Virol. 2016;88:144-52.
11. Muñoz D. Estudio piloto de la incidencia de Papilomavirus en biopsias de cuello uterino en el hospital SOLCA de Quito. Vol. 53, Journal of Chemical Information and Modeling. 2013.

12. Bedoya-Pilozo CH, Medina Magües LG, Espinosa-García M, Sánchez M, Parrales Valdiviezo J V., Molina D, et al. Molecular epidemiology and phylogenetic analysis of human papillomavirus infection in women with cervical lesions and cancer from the coastal region of Ecuador. Rev Argent Microbiol. 2018;50(2):136-46.

Received: 20 May 2019

Accepted: 2 September 2019 